\title{
Datificación en contextos de corrupción: imaginarios sociotécnicos y prácticas de resistencia frente a sistemas antipobreza en Colombia
}

\section{Carlos Barreneche ${ }^{1}$ Andrés Lombana-Bermúdez ${ }^{2}$ Juan Ramos-Martín ${ }^{3}$}

Recibido: 21/10/2020

Aprobado por pares: 03/06/2021
Enviado a pares: 13/01/2021

Aceptado: 24/06/2021

DOI: 10.5294/pacla.2021.24.3.4

Para citar este artículo / to reference this article / para citar este artigo

Barreneche, C., Lombana-Bermúdez, A. y Ramos-Marín, J. (2021). Datificación en contextos de corrupción: imaginarios sociotécnicos y prácticas de resistencia frente a sistemas antipobreza en Colombia. Palabra Clave, 24(3), e2434. DOI: https://doi.org/10.5294/pacla.2021.24.3.4

\section{Resumen}

Nuestro estudio localiza la implementación de macrodatos (big data) y la inteligencia artificial que avanza hoy en el Sur Global dentro de la agenda de "datos para el desarrollo", en culturas como las latinoamericanas, caracterizadas por grandes asimetrías sociales y una corrupción estructural -herederas del proceso de colonización-, con el fin de evidenciar los problemas de automatización en sistemas alimentados por datos que han sido históricamente objeto de manipulación, o corrupción de datos. Para ello examinamos dos casos de mal funcionamiento en infraestructuras de información de programas de asistencia social en Colombia (Ingreso Solidario y Sisben)

1 https://orcid.org/0000-0002-1784-2605. Pontificia Universidad Javeriana, Colombia. barrenechec@javeriana.edu.co

2 https://orcid.org/0000-0002-1246-5994. Pontificia Universidad Javeriana, Colombia. andresa.lombana@javeriana.edu.co

$3 \bowtie$ https://orcid.org/0000-0002-5357-8442. Pontificia Universidad Javeriana, Colombia. juanramosm@javeriana.edu.co 
y exploramos el archivo de la teoría crítica latinoamericana con el objetivo de iluminar los procesos de datificación en estas condiciones. Finalmente, nuestra aproximación responde a los llamados a cuestionar el supuesto universalismo de los datos y a ir más allá de las miradas tecnocéntricas sobre el funcionamiento de estas tecnologías, desplazando el foco de atención a los momentos en que estas fallan, a los imaginarios sociotécnicos locales y a la agencia política de aquellos que se resisten a su control normativo.

\section{Palabras clave (Fuente: tesauro de la Unesco)}

Colombia; corrupción; datificación; infraestructuras de información; pobreza; resistencia. 


\section{Datafication in Corruption Contexts: Sociotechnical Imaginaries and Resistance Practices vis-à-vis Anti- Poverty Systems in Colombia}

\section{Abstract}

Our study situates the implementation of big data and artificial intelligence, advancing today within the "development data" agenda in the Global South, in Latin American cultures characterized by marked social asymmetries and structural corruption —as heirs of colonization - It aims to highlight automation problems in systems fed by data historically subject to manipulation or corruption. For this, we examine two failures in the information infrastructures of Colombian social assistance programs (Ingreso Solidario and Sisben) and explore the archive of Latin American critical theory to enlighten datafication in these conditions. Our approach arises from calls to question alleged data universalism and go beyond technocentric views on the operation of these technologies, shifting the focus from the moments when they fail to local sociotechnical imaginary and the political agency of those who resist regulatory control.

\section{Keywords (Source: Unesco Thesaurus)}

Colombia; corruption; datafication; information infrastructures; poverty; resistance. 


\section{Dados em contextos de corrupção: imaginários sociotécnicos e práticas de resistência frente aos sistemas de combate à pobreza na Colômbia}

\section{Resumo}

Nosso estudo localiza a implementação de megadados (big data) e a inteligência artificial que hoje avança no Sul Global dentro da agenda de "dados para o desenvolvimento", em culturas como as que estão presentes na América Latina, caracterizadas por grandes assimetrias sociais e corrupção estrutural - herdadas do processo de colonização — para evidenciar os problemas de automação em sistemas alimentados por dados que historicamente foram sujeitos a manipulação ou corrupção de dados. Para isso, examinamos dois casos de mau funcionamento das infraestruturas de informação dos programas de assistência social na Colômbia (Ingreso Solidario e Sisben) e exploramos o arquivo da teoria crítica latino-americana para iluminar os processos de datificação nessas condições. Finalmente, nossa abordagem responde a apelos para questionar o suposto universalismo dos dados e ir além das visões tecnocêntricas sobre o funcionamento dessas tecnologias, deslocando o foco da atenção para os momentos em que elas falham, para os imaginários sociotécnicos locais e para a agência política daqueles que resistem ao seu controle normativo.

\section{Palavras-chave (Fonte: Tesauro da Unesco)}

Colômbia; corrupção; datificação; infraestruturas da informação; pobreza; resistência. 
Los procesos de datificación en el Sur Global han avanzado rápidamente en las últimas décadas, a medida que las naciones adelantan en el proceso de transformación digital y en la implementación de políticas económicas de convergencia de corte neoliberal y desarrollista. En el marco de estos rápidos cambios, la administración de la pobreza y los programas sociales de los gobiernos se han convertido en una de las áreas donde ha habido mayor experimentación con los sistemas basados en datos (Eubanks, 2018; Masiero y Das, 2019). El solucionismo tecnológico ha encontrado en la administración de la pobreza mediante políticas de austeridad un campo fértil para experimentar con los datos de la población más vulnerable, adelantar procesos de vigilancia masiva y automatizar la toma de decisiones.

No obstante, los problemas asociados al difusionismo y al free flow of information no son nuevos en América Latina. Ya desde finales de la década de los setenta, al calor de los debates cristalizados por la teoría de la dependencia, autores como Herrera (1973), Vaitsos (1971) y Parente (1979, en Mattelart y Schmucler, 1983, p. 12) advertían del peligro de una informatización de los Estados por transferencia directa a partir de las tecnologías, no solo de un modelo económico neoliberal imperante, sino también de una forma concreta de organización del poder de corte neocolonial, a partir de una nueva propuesta de división internacional del trabajo basada en términos cognitivos-informacionales, desarrollos infraestructurales desiguales y nuevos equilibrios geopolíticos. A estos, se sumaron voces como las de Pasquali (1976), Marques de Melo (1989) o Beltrán (1970; 1978), entre muchos otros, con un cuestionamiento surgido de las reflexiones sobre los procesos comunicacionales desde abajo, apuntando, a su vez, a la necesidad de pensar no solo una crítica al modelo desarrollista, sino un modelo propio a partir de sus mismas tecnologías, diseños, políticas y organizaciones, desde perspectivas críticas, decoloniales o posdesarrollistas que asumen, en su conjunto, la riqueza de los procesos políticos identitarios, culturales, comunitarios y horizontales de la región en la lucha por la verdadera democratización de los Estados en América Latina.

Desde los primeros intentos en Panamá de informatización, con el control de impuestos durante los años sesenta, pasando por los modelos 
Cybersyn de planificación económica en tiempo real de los trabajadores de empresas estatales en el Chile de Allende, hasta las respuestas de las dictaduras neoliberales en lo que toca a los modelos de seguridad nacional y vigilancia, paralelos a procesos de deslocalización industrial inducidos por los nuevos capitales transnacionales asociados a las telecomunicaciones durante las décadas del setenta y ochenta, la teoría crítica latinoamericana, en todas sus formas, generó un rico acervo conceptual y experiencial. Desde respuestas propositivas frente al vínculo formal entre los procesos de informatización desregulados, opacos y centralizados y el fortalecimiento de la concentración económica de las grandes empresas y el potencial control de la ciudadanía, hasta la crítica a la profundización del colonialismo y la matriz racializante de sus estructuras en Estados definidos por relaciones de corte prebendalista y clientelar.

Por tanto, este artículo inicia con un desarrollo teórico-histórico de la teoría crítica latinoamericana de la tecnología y la información, en sus múltiples formas, centrado en la crítica y análisis de los procesos de informatización de los Estados y en la manera como estos podrían derivar -en caso de asumir estrategias corporativistas de importación directa y transferencia- en valores e imaginarios directamente tributarios del imperialismo cultural, lo cual ayuda a profundizar problemáticas sociales y políticas asociadas a la pérdida de soberanía y autonomía por parte de las instituciones públicas y la ciudadanía en favor de los capitales internacionales. Tras dicho rastreo teórico-histórico, el artículo se centra en el análisis de dos estudios de caso a fin de ilustrar cómo los "diseños globales" de la datificación se sitúan en "historias locales" de adopción, oposición y disfuncionalidad (Mignolo, 2003). El primer caso se centra en el Sistema de Identificación de Potenciales Beneficiarios de Programas Sociales (Sisben), que administra la asignación de subsidios sociales en Colombia. Desde su creación en los años noventa, este programa, que otorga una puntuación a la población para determinar quién merece acceso a las ayudas sociales, ha estado envuelto en casos de corrupción: tanto de ciudadanos que intentan manipular la información que demanda el sistema como de políticos que históricamente lo han puesto a operar para el beneficio personal. En el rediseño de su última versión, el gobierno optó por implementar técnicas de macro- 
datos (big data) e inteligencia artificial integrando múltiples bases de datos públicas y privadas de acuerdo con la racionalidad de la eficiencia en la distribución de recursos, la lucha contra la corrupción y el desarrollo. El caso indaga por los procesos de datificación de la pobreza, rastreando continuidades con sistemas de clasificación sociorracial del pasado colonial, para analizar las formas en que la gente se resiste a la clasificación, la manera en que la corrupción en estos sistemas es funcional al avance del colonialismo de datos (Mejías y Couldry, 2019) y los imaginarios sociotécnicos que configuran dichas condiciones.

De forma complementaria, el segundo caso analiza una controversia pública suscitada por Ingreso Solidario, un programa de subsidios sociales que el gobierno colombiano implementó a raíz de la crisis sanitaria por covid-19, que comparte bases de datos con el Sisben. Dicha controversia se origina cuando el público puede consultar si es elegible para el programa a través del ingreso de su número de identificación en una página web del gobierno. Los ciudadanos se encontraron con diferentes tipos de errores de inclusión que serían indicativos de posibles casos de corrupción en las bases de datos del sistema. El caso analiza esta controversia explorando los imaginarios sociotécnicos que se ponen en juego en la interacción con los sistemas de información del Estado, y la auditoría ciudadana espontánea que surgió como reacción fruto de la indignación por la posible corrupción, como una forma de "resistencia algorítmica" (Treré, 2018).

\section{Teoría crítica de la tecnología latinoamericana e imaginarios sociotécnicos: datificación, corrupción y resistencias}

Los procesos de registro y control ciudadano antipobreza por parte de las instituciones estatales históricamente han mostrado numerosos sesgos derivados del carácter propiamente político y discriminatorio que se da en la base de su constitución original. Cooptados por las élites políticas y económicas de la región, los principales criterios de su avance han sido el desarrollo, la efectividad y la transparencia, que ocultan, no obstante, las relaciones de reproducción de desigualdades, patrimonialismo y corrupción presentes en sus estructuras. 
En América Latina, desde muy temprano (1570), la burocracia real se empeñó en recabar datos sobre la población en las colonias, vía interrogatorios y cuestionarios, principalmente para fines de control político y de tributación (Pérez y Montoya, 2010; Solano, 2015). De los cuestionarios que se hacían en el siglo XVI como parte de las Relaciones Geográficas de Indias a los padrones y censos del siglo XVIII, estos instrumentos de información se insertaron en un contexto marcado por una profunda corrupción burocrática. Desde los primeros momentos de la administración colonial, oficiales de todos los niveles -incluido al clero- usaron sus posiciones de poder para el enriquecimiento personal y operaron estos instrumentos para tal fin. Del impulso extractivista y rapaz de la empresa colonial emergió en el Nuevo Mundo una cultura de la corrupción que se constituyó en un dispositivo eficaz para la distribución del poder y de la riqueza entre colonizadores y élites locales (McFarlane, 1996).

En la actualidad, entre las características del capitalismo avanzado en el que se inscriben los procesos de datificación que analiza este trabajo, se incluyen en concreto la captura corporativa de las instituciones públicas en los territorios nacionales y la usurpación de la toma de decisiones políticas por parte de los poderes financieros transnacionales (Davis et al., 2020). De acuerdo con los estudios críticos de datos (Steyerl, 2019; Noble, 2018; Chun, 2019), los criterios de inclusión y exclusión que utilizan los algoritmos de reconocimiento de patrones -que emplean, entre otras, las instituciones del Estado- resultan intrínsecamente políticos e íntimamente ligados a asunciones racistas, sexistas y clasistas del propio análisis de redes (Davis et al., 2020). Sea a partir de los registros de homofilia y aislamiento en los análisis de las redes sociales digitales (Steyerl, 2019; McPherson, 2018) o del refuerzo de discriminación y segregación propio de las políticas de vigilancia basadas en registros criminales (O'Neil, 2016), desde este punto de vista, los algoritmos perpetúan la discriminación que ellos mismos encuentran (Chun, 2019) y técnicamente funcionan, por defecto, discriminando a partir de la generación de taxonomías (Benjamin, 2020; McQuillan, 2020).

En dicho proceso de menoscabo de soberanía y perpetuación de desigualdades, las instituciones estatales ven debilitado su poder, hasta perder 
el control político ejercido por los Estados-nación en pos de los poderes corporativos y crearse una sensación entre la ciudadanía de pérdida de soberanía, deslegitimación y necesidad de lucha conjunta frente al sistema capitalista y el Estado, de forma integrada.

Una de las principales consecuencias de dicha deslegitimación es el aumento de la preocupación general por parte de la ciudadanía acerca de la gravedad de los procesos de corrupción (Rivera, Plata y Rodríguez, 2018). Precisamente, el último Barómetro de las Américas para Colombia, del año 2018, muestra una creciente preocupación por la corrupción, tanto a nivel macro (participación de políticos y funcionarios públicos con capacidad de toma de decisiones) como a nivel micro (que implica la cotidianidad de la participación de la ciudadanía en busca de condiciones más favorables), hasta alcanzar dicha percepción su valor histórico más alto (19\%, por encima del conflicto armado). No obstante, esta impresión no solo engloba a la clase política, sino también se extiende a la propia ciudadanía. En ese sentido, son la decepción con el sistema político, unida al miedo a las represalias, la inacción o la propia necesidad, las causas que sirven de motivación para participar en procesos de microcorrupción ciudadana (Rivera et al.,2018).

Rastreando las razones de la corrupción institucional en los sistemas políticos latinoamericanos, esta posee, en la gran mayoría de las ocasiones, una raíz histórico-estructural imbricada en procesos donde tienen gran relevancia el patrimonialismo, el autoritarismo, el rentismo, la dependencia extractivista, la falta de protagonismo de los movimientos sociales y la incapacidad de los gobiernos para transformar las relaciones heredadas y los Estados en América Latina (Lynch, 2020).

Es precisamente en la relación entre corrupción y extractivismo donde se encuentra, desde una perspectiva decolonial (Gudynas, 2017; Lander, 2004), una posible explicación para el desarrollo de inequidades en la interacción gobierno-empresa, al establecer mecanismos de compensación para la eficiencia inscritos en los procesos de la administración pública en América Latina. Preocupados siempre por la profundización de modelos desarrollistas, lejos de ofrecer ventajas comparativas frente a las condiciones de la organización política de los Estados, el modo de funcionamiento 
de los sistemas políticos (descritos, a grandes rasgos, como regímenes delegativos con alta carga de hiperpresidencialismo en interacción con capitales privados) favorece procesos de cooptación, tráfico de influencias y soborno que profundizan el esquema de desigualdades. De hecho, es en la propia concepción del "desarrollo" donde la corrupción está más presente, en la medida en que ella comporta un "núcleo básico de concepciones, como el progreso material y económico basado en la explotación de la Naturaleza, la obsesión con el crecimiento económico, la rentabilidad y el individualismo competitivo", todo lo cual "abre muchos flancos para que penetre la corrupción" (Gudynas, 2017, p. 85).

En ese sentido, la teoría latinoamericana, desde hace más de 50 años, se viene cuestionando la relación entre desarrollo tecnológico, informatización de los procesos de registro y control por parte del Estado y la incursión del modelo corporativo en la toma de decisiones públicas como razones de la profundización de los problemas socioeconómicos de la región. Con una mirada propia y atendiendo a los problemas específicos de las realidades latinoamericanas, las críticas desarrolladas y sus propuestas posteriores han buscado la construcción de una perspectiva regional en lo concerniente a las implicaciones sociales, económicas, políticas y culturales de la integración de nuevas tecnologías de información y comunicación en la constitución de los procesos de interacción y funcionamiento del Estado en busca del desarrollo. A partir de los primeros análisis críticos del papel de las infraestructuras de información en América Latina en los procesos de informatización de los Estados, se divisan, al menos, dos corrientes que aportan una conexión directa con los postulados propuestos por la teoría de la dependencia (Cardoso y Faletto, 1969; Gunder, 1971) y perspectivas críticas afines (Prieto, 1988).

Por una parte, se encuentra el análisis aportado por los estudios críticos de economía política de información, comunicación y cultura (Mattelart y Schmucler, 1983; Beltrán, 1970; 1978) y la Escuela Latinoamericana de Comunicación, centrados muy especialmente en el papel de las infraestructuras y los procesos de transferencia y en la construcción de desigualdades a partir de la asimilación de formas de dominación e imperialismo 
cultural (Beltrán, 1970; Prieto, 1988). Dichos procesos generaron la conciencia de una dependencia doble: por un lado, interna, en el control por parte de las élites urbanas, conectadas con las corporaciones transnacionales, sobre el resto de la población local; y por otro, externa, en forma de imperialismo cultural e imposición de una serie de imaginarios sociotécnicos (creencias, mitos, valores y normas) importados desde las realidades del Norte a las del Sur Global, a través de esquemas verticales de persuasión y procesos de monopolización de los sectores informacionales y comunicacionales por parte de capitales corporativos transnacionales (Mattelart y Schmucler, 1983; Pasquali, 1976; Marques, 1989).

Por otro lado, se encuentra la corriente de Pensamiento Latinoamericano en Ciencia, Tecnología y Sociedad (Placts), definida durante los años sesenta y setenta en la región y muy cercana también a los postulados de la teoría de la dependencia (Dagnino, Thomas y Davyt, 1996), la cual ayuda a comprender los orígenes causales del avance de la problemática actual en los procesos de datificación, a partir de una explicación que vincula, de nuevo, la informatización, el avance del capitalismo y el cambio en el rol de intervención política por parte de los Estados (Herrera, 1973; Vaitsos, 1971). Desde una perspectiva crítica pragmática, la Placts proponía la configuración de un "mix tecnológico" adecuado a las condiciones locales y señalaba la necesidad de construir procesos de acumulación de saber propios en relación con la selección de tecnologías acordes. A partir de dichos procesos iniciales, se podría entonces establecer una soberanía tecnológica nacional dada por la integración del desarrollo tecnológico en un proyecto nacional determinado (Dagnino et al., 1996).

Finalmente, en el desarrollo de la interpretación de las resistencias a los modelos de imperialismo y dominación tecnológica, el concepto de "imaginarios" resulta útil para entender la forma en la que los sistemas de información que utilizan big data e inteligencia artificial son experimentados por las poblaciones en contextos de corrupción en el Sur Global y los discursos y acciones que surgen de esas situaciones. Desde las ciencias sociales, los imaginarios han sido entendidos como esquemas mentales socialmente construidos, representaciones simbólicas de la realidad que tienen im- 
plicaciones políticas y sirven para unificar y ordenar la sociedad influyendo en las acciones individuales y colectivas (Baczko, 1991; Castoriadis, 2013; Pintos, 2015; Taylor, 2004). La fuerza de los imaginarios (p. ej. normas, valores, orientaciones morales) se encuentra precisamente en la forma en que son actuados y mantenidos por colectivos e instituciones, así como en sus implicaciones políticas (Castoriadis, 2013).

Particularmente para nuestro análisis de las experiencias de datificación en el contexto colombiano, nos enfocamos en la noción de imaginarios sociotécnicos. De acuerdo con Jasanoff (2015a), los imaginarios sociotécnicos son visiones colectivas de futuros deseables, articuladas a partir de entendimientos compartidos de formas de vida y orden social, que son alcanzables a través de, y apoyando a, el avance de la ciencia y la tecnología. Estos imaginarios ocupan una zona híbrida entre lo mental y lo material. Son coproducidos colectivamente no solo a través de los discursos que actores sociales, en diferentes posiciones de poder, circulan en la sociedad en relación con la ciencia, la tecnología y el futuro colectivo deseado, sino también por medio de las acciones performativas (prácticas) que los actores realizan, y a través de su materialización en sistemas tecnológicos y la conformación de instituciones. Por consiguiente, los imaginarios sociotécnicos tienen una dimensión performativa y de acción y son al mismo tiempo productos e instrumentos en la coproducción de ciencia, tecnología y sociedad (Jasanoff, 2015a).

Debido a que existe una competencia entre grupos y actores sociales por establecer sus visiones de futuros deseables a partir de sus propios entendimientos del orden social y formas de vida, diferentes imaginarios sociotécnicos pueden coexistir de forma simultánea. Siguiendo a Jasanoff (2015b) y a Kim (2015), reconocemos la existencia de dos tipos de imaginarios sociotécnicos: dominantes y alternativos. Mientras algunos imaginarios sociotécnicos son establecidos como dominantes por actores estatales y no estatales que cuentan con recursos económicos y autoridad política para dar sentido de orden a la sociedad y legitimar ciertos usos y desarrollos de la ciencia y la tecnología, otros imaginarios permanecen como alternativos y son articulados por actores de la sociedad civil como activistas, orga- 
nizaciones no gubernamentales y ciudadanos del común. Los imaginarios sociotécnicos alternativos son construidos en oposición a los dominantes, desafiando las prioridades, privilegios y visiones de futuro preformados desde el Estado y sus instituciones oficiales (Kim, 2015). Debido a su carácter contestatario y de oposición, podemos considerar estos imaginarios sociotécnicos alternativos como imaginarios de resistencia.

En el análisis de los procesos de datificación, investigaciones recientes han utilizado el concepto de imaginarios sociotécnicos precisamente para entender cómo los ciudadanos y comunidades dan sentido a sus prácticas y experiencias con datos y algoritmos (Bucher, 2017; Kazansky y Milan, 2021; Lehtiniemi y Ruckenstein, 2019; Mansell, 2012). El reciente trabajo de Kazansky y Milan, por ejemplo, presenta tres casos de proyectos de software libre y contravigilancia (Qubes OS, Dowse y CV Dazzle) para ilustrar la existencia y potencial de imaginarios sociotécnicos alternativos. Denominándolos “contraimaginarios", las investigadoras destacan el papel movilizador de imaginarios que, en oposición a los imaginarios dominantes sobre la datificación construidos por el Estado y las corporaciones, logran animar respuestas tácticas e intervenciones técnicas por parte de la sociedad civil para mitigar los daños y amenazas de las tecnologías de vigilancia masiva.

\section{Metodología}

Nuestro enfoque metodológico es múltiple e interdisciplinario, combinando la reflexión crítica, histórica y teórica sobre la datificación en contextos del Sur Global con la investigación empírica e interpretativa sobre los imaginarios sociotécnicos y las prácticas de resistencia en los programas Ingreso Solidario y Sisben en Colombia. Utilizando esta aproximación mixta elaboramos dos casos de estudio para revelar las particularidades de la implementación de big data e inteligencia artificial en contextos de desigualdad y corrupción estructural como los de la mayoría de países latinoamericanos. Específicamente, esta metodología nos permite ilustrar la forma en que los sistemas de información disfuncionales son promovidos por imaginarios sociotécnicos dominantes y resistidos a la vez por imaginarios alternativos. 
En la elaboración de los dos casos de estudio hemos utilizado métodos cualitativos para analizar fuentes primarias y secundarias relacionadas con los programas Ingreso Solidario y Sisben que incluyen: documentos de políticas públicas, documentos técnicos, investigaciones académicas y de organizaciones internacionales, noticias, comunicados de prensa del gobierno y comentarios de ciudadanos y ciudadanas en redes sociales (Twitter y YouTube). Además, hemos utilizado métodos computacionales para recolectar y analizar datos de la controversia pública desarrollada en Twitter sobre Ingreso Solidario durante la primera etapa de su implementación. Específicamente el uso de los software Nvivo y DMI-TCAT (Borra y Rieder, 2014) ha apoyado nuestro proceso de captura y organización de datos, análisis de contenido, codificado sustantivo y mapeo de imaginarios.

El estudio de caso sobre la primera etapa del programa Ingreso Solidario abarca el periodo de tiempo que va del 7 de abril de 2020 (lanzamiento del programa) al 22 de abril de 2020 (reactivación del programa e inicio de la etapa 2) y se concentra en la discusión pública que tuvo lugar en la red social Twitter. Los datos fueron recolectados utilizando la herramienta DMI-TCAT. Capturamos todos los tuits públicos que contenían los hashtags: CorrupcionConLasAyudasEs, CorrupcionSolidaria, FraudeDeEstado, IngresoSolidario, IngresoSolidarioCorrupto, RoboSolidario, y también aquellos que mencionaban al usuario @DNP_Colombia. Este proceso nos permitió identificar la red temática de tuits que se generó durante la controversia, la cual se compone de 88.416 tuits públicos, enviados por 38.559 usuarios diferentes durante el periodo de tiempo que va del 9 al 22 de abril de 2020 .

Para el estudio de caso del Sisben abarcamos el periodo 2016-2020, que comprende la etapa de evaluación, rediseño e implementación de la nueva versión del sistema (Sisben IV). Nos centramos en el análisis de fuentes secundarias, como el estudio de grupos focales de beneficiarios contratado por el Estado (DNP, 2016a), y primarias, como los documentos oficiales del gobierno y los comentarios públicos de ciudadanos en videos de YouTube sobre el sistema. 
Vale la pena anotar que, dadas las características de los ciudadanos y ciudadanas colombianas que participan en las redes sociales, los imaginarios que hemos mapeado en nuestro análisis presentan sesgos y corresponden a poblaciones específicas. En la red social Twitter, estos imaginarios son articulados desde la perspectiva de una población que es en su mayoría urbana, adulta, de género masculino y con niveles de educación medios y altos. En contraste, los imaginarios que se visibilizan en YouTube provienen de una población más diversa en cuanto a edad, género, ubicación geográfica y nivel educativo. ${ }^{4}$

Dado el carácter público de las publicaciones en Twitter y en YouTube, con su disponibilidad, facilidad de acceso y relevancia para el discurso y la opinión pública, estas plataformas han sido utilizadas por investigadores de diferentes disciplinas para estudiar diversas problemáticas, tales como el discurso en línea, los movimientos sociales y la circulación de información en los ecosistemas mediáticos. Los datos de Twitter que hemos utilizado para construir uno de los casos de estudio los hemos capturado respetando las políticas de privacidad, los términos y condiciones de la plataforma y los derechos de los usuarios y utilizando la API (interfaz de programación de aplicaciones) que está disponible públicamente. En la elaboración de los dos casos hemos decidido mantener los nombres que los usuarios utilizan en las plataformas porque consideramos que sus tuits y comentarios son públicos y, al darles el crédito como creadores de estos contenidos, al mismo tiempo respetamos sus derechos como autores y situamos los imaginarios sociotécnicos en ciudadanos específicos.

\section{Imaginarios sociotécnicos y prácticas de resistencia en el Sisben e Ingreso Solidario}

Los dos casos de estudio que presentamos a continuación son complementarios, en la medida en que tanto el Sisben como el programa Ingreso Solidario son sistemas para la administración de la pobreza basados en big data e inteligencia artificial implementados en Colombia en las últimas dé-

4 De acuerdo con el Digital 2020 Global Overview Report, YouTube es la red social con mayor número de usuarios en Colombia, con 30,1 millones, mientras que Twitter tiene 3,2 millones (Medina 2020). 
cadas. El primero se desarrolló desde la década de los noventa del siglo pasado, con diferentes iteraciones, y el segundo fue implementado en 2020 durante la pandemia de covid-19. Ambos casos están intrínsecamente relacionados, no solo en sus objetivos, sino también en sus diseños técnicos y sistemas de clasificación. El programa Ingreso Solidario, por ejemplo, hace uso de las bases de datos creadas por el Sisben (III y IV) para identificar ciudadanos en condiciones de pobreza y vulnerabilidad que recibirán un subsidio monetario.

A continuación, se presentan los análisis diferenciados, a fin de evidenciar las conexiones, similitudes y diferencias entre ambos casos, así como en las prácticas de resistencia e imaginarios sociotécnicos presentes.

\section{Una venganza ancestral: corrupción y mal funcionamiento en el Sisben}

Como respuesta a la crisis económica y social de los años ochenta en América Latina, y en el marco de políticas de austeridad derivadas del Consenso de Washington, los países de la región abandonaron el enfoque de cobertura universal de las políticas sociales y optaron en su lugar por una estrategia de focalización del gasto social guiada por la racionalidad burocrática de la eficiencia. En Colombia, desde 1994, se implementó el Sistema de Identificación de Potenciales Beneficiarios de Programas Sociales (Sisben), un sistema de información socioeconómica para la focalización de los programas sociales del Estado. El sistema de clasificación comprende el cálculo de un índice del bienestar económico de los ciudadanos (puntaje del Sisben), que determina quiénes merecen acceder a las ayudas sociales a partir de la administración de una encuesta que recoge datos de las características observables de los hogares y la condición socioeconómica de las personas que los conforman. El Sisben se ha constituido así en una clasificación crítica para la supervivencia de millones de ciudadanos en condiciones de vulnerabilidad que de otra forma no tendrían la posibilidad de acceder a servicios básicos.

Actualmente el gobierno está completando la implementación de la cuarta versión del sistema (Sisben IV). El nuevo diseño está orientado a ex- 
tender el proceso de datificación de la población (la meta es incluir al 80\% de toda la población, ampliándolo a otros sectores sociales) con el fin de corregir los problemas de calidad de información-llamados errores de inclusión-, que se le han atribuido principalmente a la acción de los llamados "colados" (personas que logran clasificar al acceso a las ayudas engañando al sistema). Para este fin, se instauró, a través de alianzas público-privadas, un sistema de verificación que comprende cruces de información entre 34 bases de datos públicas y privadas para identificar inconsistencias en los datos y así filtrar a los colados de los "verdaderos" pobres, legítimos merecedores de las ayudas (Conpes, 2016). Adicionalmente, se introdujeron cláusulas que obligan a asumir responsabilidades legales a aquellos encuestados que entreguen información falsa o incompleta, con la amenaza de perder el acceso a los servicios esenciales. Así, la extensión de la datificación en nombre de la lucha contra la corrupción permite al gobierno hostigar y castigar a los pobres (Eubanks, 2018) a través de la institución de una "moralidad técnica” (McQuillan, 2020) de depuración de los datos.

Como parte de estos acuerdos, se enlistó también como socio a $E x$ perian -agencia de crédito $y$ data-broker multinacional- para introducir una modificación en el algoritmo que establece los puntajes, con el fin de incluir estimados de la capacidad de ingreso de las personas e identificar "falsos" pobres o pobres que podrían dejar de serlo en el futuro. Con una lógica de gobierno algorítmico de tipo inferencial (predicción-prevención) que incorpora las técnicas de analítica de datos del sector de FinTech para detectar fraudes financieros a través del cruce de bases de datos, y la identificación de clientes rentables con capacidad de pagar créditos, el ciudadano deja de concebirse como un sujeto de derechos para convertirse en el sujeto de riesgo de las finanzas y, subsecuentemente, en consumidor (vía bancarización) y mercancía cuando sus datos son compartidos y explotados a través de alianzas público-privadas para el desarrollo de productos financieros para los bancos y otros emprendimientos (Fundación Karisma, 2020). De esta manera, la solución para corregir los errores de inclusión -esto es, lograr la exclusión de más personas del sistema- es presentada como una política de inclusión social dentro de un marco discursivo desarrollista: democratización del acceso al sistema financiero y fomento del 
emprendimiento (Fundación Karisma, 2020; Arora, 2016). Cuando el acceso a derechos está condicionado por el consentimiento a la vigilancia y mercantilización de los datos personales, se configura así una forma de inclusión depredadora de los vulnerables, un modo de colonialismo interno de datos (Ricaurte, 2019).

El rediseño del Sisben responde a una estrategia de vigilancia de datos que busca la detección del colado como principio (Fundación Karisma, 2020) y que es justificada como cruzada moral anticorrupción: "El hecho de que estén aumentando de manera tan importante los colombianos de altos ingresos que se cuelan en los programas sociales es supremamente preocupante, indignante, inaceptable [...] porque no hay corrupción buena, [y] que se permita este tipo de ejercicio no es justo con los colombianos humildes" (DNP, 2016).

En el discurso de indignación del gobierno se presenta una imagen del colado como alguien que, por un lado, no es "merecedor" de las ayudas (error de inclusión) y, por otro lado, además es responsable de que los pobres no accedan a ellas (error de exclusión). La condena moral de los colados individualiza asíla responsabilidad del mal funcionamiento del sistema, proyectándose en la corrupción de ciudadanos particulares, mientras produce el efecto ideológico de despolitizar la pobreza, al desplazar la responsabilidad del Estado como garante de derechos y su obligación de abordar las condiciones estructurales que la reproducen.

Sin embargo, la percepción de aquellos que participan de la medición por necesidad es otra. En los imaginarios de los sisbenizados, tal cual se expresan en los comentarios de los videos en Youtube, "el sisben es una fachada de la corrupción” del gobierno (Quintian, citado en William Cardona, Canal Oficial, s.f.), donde políticos y funcionarios públicos son los principales beneficiarios de los errores de inclusión. Gracias a la autonomía y falta de vigilancia que tienen los funcionarios encargados de registrar la información, son prácticas comunes de los políticos regionales el ofrecer la vinculación al sistema como beneficiarios a cambio de votos (clientelismo), el establecimiento de mercados ilegales donde se venden los cupos o 
la apropiación de parte de los recursos destinados a esas ayudas (p. ej. registrando personas fallecidas), entre otras prácticas de corrupción. A partir de estos imaginarios los colados justifican sus propias prácticas: "Si los de arriba roban, porque nosotros no" (Arciniegas, citado en William Cardona, Canal Oficial, s.f.); "si los ricos lo hacen sin necesidad, mas (sic) por la necesidad lo hacen los pobres así parezca injustificable" (Molina, citado en William Cardona, Canal Oficial, s.f.).

Existe una queja generalizada en los usuarios del sistema por la mala asignación de la clasificación, al considerar que esta no refleja sus condiciones materiales reales de existencia, mientras cualquier mejora de las mismas es castigada con la exclusión de los beneficios, en lugar de ser incentivada (DNP, 2016a). Por ejemplo, y gracias a la opacidad en que se mantiene la forma en que se construyen estas clasificaciones, en los imaginarios sociotécnicos populares existe la creencia de que un mejoramiento en la infraestructura de los barrios y lugares de habitación puede derivar en una penalización en la clasificación, por lo que muchos prefieren mantener las condiciones de precariedad para conservar las ayudas. Igualmente, algunos se ven obligados a optar por trabajos informales o por servicios prepago de telecomunicaciones para evitar el registro en las bases de datos de verificación. De este modo, a través de una infraestructura de información defectuosa y excluyente por diseño, el Estado interviene produciendo marginalización (Acevedo-Guerrero, 2019).

La trampa es de hecho una práctica extendida entre aquellos que participan de la medición por necesidad: "hay que hacer trampa y ser deshonestos para recibir un auxilio" (sisbenizado, citado en DNP, 2014, p. 76), y esta se manifiesta reactivamente como tácticas de resistencia a la exclusión por diseño del sistema. Asumiendo la apariencia de obediencia frente a la medición, el repertorio de prácticas populares se despliega frente al encuestador como una performance de la miseria a través de tácticas miméticas (imitación de la señal) (p. ej. ocultar electrodomésticos y artículos de lujo, convocar más personas para presentarlas como parte del núcleo familiar, simular vivir hacinados en un cuarto rentado, hacerse pasar por desplazado, etc.), apelando a la "desinformación constructiva" -información 
falsa que, en lugar de apuntar al sabotaje del sistema (ruido), tiene por objetivo inducirlo al error, al ser registrada como verídica (señal) (Alexander y Smith, 2016) - (p. ej. mentir sobre la condición de empleo, ingresos, nivel de escolaridad, exagerar la cantidad de hijos, etc.). Estas tácticas de desobediencia disimulada de la norma exponen a la vez la fragilidad del poder regulador del Estado, materializada en el mal funcionamiento de la infraestructura de información, al que este responde luego con un nuevo rediseño para solucionar los "errores" deliberadamente provocados por estas prácticas, en un ciclo de mutua constitución.

A estas actitudes de astucia, invención y burla frente al poder se las ha caracterizado popularmente en Colombia como "malicia indígena". Este es un concepto que connota a la vez un antivalor, como expresión racista de desprecio por un supuesto carácter tramposo del indígena, y un valor, reivindicado en el imaginario popular como mecanismo adaptativo que permite al subalterno lograr alguna ventaja o superar al otro en condiciones asimétricas de poder: "Desde afuera es juzgado como indisciplina, exclusivamente, pero al interior mismo del valor, es la puesta en marcha de actitudes necesarias y compartidas, para conseguir fines prácticos, con un doble respaldo: el que los de mayor poder también lo hacen y la inoperancia de la norma y de las instituciones de autoridad, que precisamente son partícipes del valor mismo" (Morales, 1998).

Las raíces de la malicia indígena se encuentran en las tácticas de los pueblos amerindios oprimidos para enfrentar los procesos de colonización (Rappaport, 2014; Pérez, 2016) y su persistencia en las sociedades mestizas contemporáneas -como fantasma de una venganza ancestral- es un "testimonio de resistencia a largo plazo y de justicia” (Morales, 1998).

Al igual que en el caso del Sisben, los intentos de medición y clasificación sociorracial en la Colonia fueron un lugar de disputa y tensión social, cuya implementación estuvo marcada por una profunda desconfianza entre los agentes que administraban los registros y aquellos que eran sometidos a los mismos, y fueron un objeto de cuestionamiento permanente de sus resultados y eficacia (Rappaport, 2014; Solano, 2015). Por ejemplo, el 
virrey Antonio Caballero y Góngora se refería así a las razones del fracaso de los primeros censos del siglo XVIII:

... por mucho cuidado que se quiera poner en la formación de un
padrón general, jamás se alcanzarán a comprender las ocultas ran-
cherías [...], la malicia de algunos y la ignorancia de los más hizo ex-
tender la voz de que esta operación era dirigida a un nuevo impuesto,
con lo que los padres ocultaban la mitad de su familia si no podían
esconderse en los montes mientras el empadronamiento. (Colmena-
res, 1989, p. 404)

Además de intentos como estos de evadir la cuantificación, otra práctica extendida entre aquellas gentes que los españoles calificaban como maliciosas e indisciplinadas comprendía aprovecharse de la categoría sociorracial de mestizo en las taxonomías de estos instrumentos con el fin de evadir impuestos. Como lo demuestra Joane Rappaport (2014), en la Nueva Granada de los siglos XVI y XVII ser mestizo no constituía una identidad social -ningún grupo se reconocía a sí mismo como mestizo- $y$, más bien, la categoría fue inicialmente una construcción administrativa, explotada por los súbditos de la Corona como una "estrategia de identificación". Aprovechando los bordes borrosos entre las categorías indio, mulato y mestizo, la gente usaba su apariencia para burlar los defectuosos instrumentos fisionómicos de identificación racial -importados desde Europa y adaptados sin éxito a las complejas condiciones sociorraciales locales del mestizaje-, con el objetivo de ser clasificada en otra categoría, dedicando un gran empeño en frustrar y controvertir la clasificación hasta donde el aparato legal se lo permitía (Rappaport, 2014).

La malicia indígena es pues una expresión de los modos de resistencia que despliegan aquellos que viven en condiciones históricas de asimetría social y corrupción, herederas del colonialismo, y una ilustración de la inventiva y recursividad a la que apelan quienes son coaccionados por necesidad a la inclusión en sistemas que automatizan la distribución de recursos escasos (austeridad), cuando no está disponible la opción de no participar de los mismos (opting-out) o de limitar su acción nociva apelando al derecho a la privacidad o a las tácticas de invisibilidad del anonimato. De manera similar a la estrategia de identificación como mestizo en los 
padrones coloniales, la malicia indígena, como modo de resistencia algorítmica, se manifiesta en las prácticas de los colados como un recurso a la desinformación para inducir cierta clasificación: pasar por otra categoría para sacar ventaja de un sistema adversario, un "ataque adversario" del que se resiste a la exclusión.

El caso del Sisben expone así cómo aquellos en condición de vulnerabilidad no son simples objetos pasivos de la cuantificación y muestra que, a pesar de las limitaciones de agencia de su localización social, se resisten de varias formas a la clasificación, partiendo de sus repertorios culturales. Finalmente, el caso también muestra cómo los intentos de datificación son parte de luchas sociales que se concretan en los mismos diseños de las infraestructuras de información, donde, además de la materialización y automatización de ciertos valores morales y políticos en las estructuras de datos y en los algoritmos (Bowker y Star, 2000; Eubanks, 2018), se incorporan también, de forma reactiva, las tácticas de resistencia ciudadana como innovación tecnológica en los diseños, con lo cual se evidencia el carácter político y coproducido de estas tecnologías, una vez son adaptadas a los contextos del Sur Global.

\section{Auditoría colectiva de Ingreso Solidario}

El 7 de abril de 2020, el gobierno colombiano inició la implementación de la primera etapa de Ingreso Solidario, un programa social de transferencias monetarias a la población vulnerable y en condición de pobreza (aproximadamente, tres millones de ciudadanos), creado como medida de emergencia durante la crisis por el covid-19. El programa hace uso de big data (varias bases de datos públicas y privadas) y tecnologías de información para identificar a las personas en condición de vulnerabilidad y transferirles un subsidio de $\$ 160.000$ pesos. ${ }^{5}$ Desarrollado en medio de la crisis ori-

5 En el "Manual operativo del Programa Ingreso Solidario" (DNP, 2020) se menciona la creación de una Base Maestra como resultado de cruzar más de 10 bases de datos y registros de programas del gobierno, incluidas las del Sisben III (certificada) y Sisben IV (consolidada), y de la utilización de "un algoritmo fonético que aumenta la probabilidad de éxito de asignar la información de una persona de una base a otra. En especial, se tienen en cuenta los números de identificación, los nombre y apellidos y las fechas de nacimiento". El manual menciona también el uso de bases de datos (privadas) de entidades financieras reportadas a la central de información TransUnion, las cuales serán cruzadas con la base maestra para verificar a los potenciales beneficiarios. Además, para el caso de beneficiarios no bancarizados, en el manual se explica que el DNP remitirá a los Proveedores de Redes y Servicios de Telecomunicaciones 
ginada por la pandemia y en el marco del estado de emergencia económica, social y ecológica declarado por el gobierno, el programa fue planeado y diseñado en pocas semanas y presentado como una iniciativa innovadora y de gran esfuerzo técnico. "En este esfuerzo sin precedentes y en tiempo récord, el Gobierno del Presidente Duque contó con el acompañamiento de las entidades financieras, las centrales de riesgos y las empresas de telefonía celular, para facilitar el contacto con los hogares beneficiarios", declaró la Presidencia de la República en un comunicado oficial.

El subdirector del Departamento Nacional de Planeación (DNP), institución encargada de diseñar e implementar la metodología de focalización de los potenciales beneficiarios y de consolidar y administrar la "base de datos maestra", describió el programa como innovador, enfatizando el cruce de "muchas bases de datos para llegar al mayor número de colombianos posibles". El funcionario del gobierno explicó: "hemos venido refinando las bases de datos. Hoy tenemos un sistema actualizado que tiene información cada vez más realista sobre la situación de focalización" (Presidencia de la República, 2020).

El lanzamiento del programa Ingreso Solidario incluyó la apertura de una página web oficial que permite realizar consultas de números de cédulas de ciudadanía (números de identificación nacional) a través de un buscador. Es precisamente la interacción de ciudadanos y ciudadanas con el buscador y el encuentro con errores de inclusión en los resultados de búsqueda lo que desencadenará una auditoría colectiva al sistema de información de Ingreso Solidario y alimentará la controversia sobre la corrupción del programa en la esfera pública, principalmente en Twitter. Al introducir números de identificación para saber si han sido elegidos como beneficiarios del programa, los ciudadanos descubren errores de inclusión e inconsistencias en los resultados de sus búsquedas. Mientras muchos ciudadanos encuentran que han sido excluidos del programa, a pesar de sus condiciones de pobreza y vulnerabilidad, otros descubren que muchos de los beneficiarios tienen números de identificación de personas inexistentes o muertas,

(Comcel, Telefónica, Colombia Móvil, Avantel, ETB, Virgin, Flash Mobile, Móvil Éxito y Suma) la base de datos maestra para que estas empresas identifiquen a los beneficiarios y puedan ser contactados. 
mientras que varios de ellos aparecen asociados a un solo número de identificación. Estas acciones pueden entenderse como experiencias de datificación donde los ciudadanos pueden experimentar, de forma directa, en tiempo real y con la mediación de dispositivos digitales (computadores y teléfonos móviles), fallos en un sistema de información diseñado para administrar la pobreza en tiempos de pandemia.

La discusión generada en Twitter durante la primera fase de implementación del programa Ingreso Solidario se alimenta del choque entre diferentes imaginarios sobre las tecnologías de información del Estado colombiano. Por un lado, encontramos el imaginario dominante de actores institucionales, tecnócratas, políticos y ciudadanos del partido del gobierno que articulan un discurso que defiende el programa Ingreso Solidario por su eficiencia técnica y niega que sea un caso de corrupción. Por otro, encontramos un imaginario alternativo articulado por políticos de partidos de oposición al gobierno, activistas y ciudadanos del común a través de un discurso que critica el programa Ingreso Solidario relacionándolo con la corrupción, el fraude, la trampa y el robo y asociándolo con un problema sistemático de disfuncionalidad y mal funcionamiento de todos los sistemas de información del Estado. ${ }^{6}$

A partir del encuentro con errores de inclusión al utilizar el buscador en la página web de Ingreso Solidario, cientos de ciudadanos comienzan a publicar (el día siguiente a la implementación del programa) contenidos en Twitter denunciando la corrupción del gobierno y de las instituciones del Estado. Como respuesta a las denuncias, el DNP realiza dos publicaciones en Twitter el 8 de abril en las que explica que se "se presentó una anomalía con el buscador de cédulas" y que "la función de búsqueda en la página web es independiente y no afecta los giros a beneficiarios". El discurso articulado por el DNP en estas publicaciones hace parte de un imaginario que

6 Cabe anotar que esta controversia se desarrolló en medio de un contexto de crisis política caracterizado por la protesta social y una intensa crítica al gobierno de Iván Duque (agosto de 2018 - presente) por parte de múltiples sectores, incluidos políticos de oposición, estudiantes, ONG y activistas. En un abanico que comprende desde intensas discusiones sobre la legitimidad del gobierno y sus funcionarios -que incluyeron denuncias sobre sus nexos con el narcotráfico, la compra de votos en elecciones y los contratos amañados-, hasta numerosas protestas sociales masivas (paros nacionales) en las calles de las principales ciudades (noviembre a diciembre de 2019), el clima de crisis ha alimentado hasta el presente el activismo político de oposición al gobierno en espacios públicos en línea y fuera de línea. 
presenta el programa Ingreso Solidario como un sistema eficiente, funcional, con impacto en millones de personas en condición de vulnerabilidad (beneficiarios). Sin embargo, este imaginario minimiza los errores de inclusión experimentados por los ciudadanos, restándoles importancia y desvinculándolos de las transferencias de dinero. La explicación dada por el DNP, y también por varios tecnócratas y miembros del partido del gobierno, es cuestionada por muchos ciudadanos, políticos de oposición y activistas. El imaginario sociotécnico dominante es motivo de indignación y hace que imaginarios alternativos sobre el sistema de información de Ingreso Solidario y sus diferentes componentes (bases de datos -incluyendo las del Sisben-, algoritmo, software, servidores web, aplicativos, entre otros) sean articulados y visibilizados desde posiciones subalternas, marginales y de resistencia.

El esfuerzo de ciudadanos por explicar los errores de inclusión se torna rápidamente en una tarea colectiva, la cual es coordinada de forma descentralizada a través del uso de hashtags como \#FraudeDeEstado, \#RoboSolidario, \#IngresoSolidarioCorrupto $\mathrm{y}$ \#laCorrupcionEselPeorVirus que son fáciles de reconocer y sintetizan, por su brevedad, un imaginario vernáculo sobre tecnologías de información corruptas. Estos hashtags son estratégicamente utilizados por ciudadanos como parte de un proceso de resistencia algorítmica (Treré, 2018) y de "protesta social hashtag" (Jackson, Bailey y Foucault, 2020) que puede entenderse como una auditoría pública y popular. Cientos de ciudadanos participan en la auditoría pública aportando evidencias (textos, capturas de pantalla, videos) sobre sus interacciones con el buscador y, de esta forma, con base en la exposición colectiva de errores de inclusión experimentados en el sistema, articulan un imaginario alternativo que relaciona las tecnologías de información con la corrupción.

A medida que la discusión en Twitter crece y los hashtags se sitúan como tendencias en Colombia, la controversia sobre Ingreso Solidario es abordada por los medios de comunicación tradicionales, quienes hacen uso de las evidencias recolectadas y compartidas por cientos de ciudadanos en Twitter para construir notas periodísticas sobre el escándalo de corrupción. Este fenómeno, como bien explica Treré (2018), refleja la capacidad 
de los ciudadanos para explotar los algoritmos de las redes sociales e influir en la agenda de los medios de comunicación. La apropiación del algoritmo de Twitter para coordinar de forma descentralizada una auditoría colectiva al sistema de información de Ingreso Solidario demuestra además la confluencia de la resistencia algorítmica con la resistencia a la corrupción en el contexto local colombiano.

Un día después de que la controversia se ha tomado la esfera pública, el gobierno desactiva la función del buscador en la página web oficial de Ingreso Solidario (9 de abril). Esta situación abre la posibilidad para que los ciudadanos que participan en la auditoría colectiva describan componentes específicos del sistema de información como su página web, la aplicación de búsqueda y su base de datos. Además, motiva a que ciudadanos con conocimientos de ingeniería se sumen a la auditoría no solo compartiendo información técnica, sino también realizando intervenciones en el sistema para reconectar la función del buscador en la página web y poder continuar con el proceso de indagación. Por ejemplo, uno de los hilos más populares (más de 8.000 retuiteos) es el que realizó un ingeniero de sistemas. El hilo encadena 29 tuits en los que, por medio de frases cortas y capturas de pantalla (de la página web, de los resultados de búsqueda y de diferentes secciones del código fuente), el ciudadano explica el funcionamiento de los diferentes componentes del sistema y así mismo enfatiza que los errores en los resultados no son por una falla en el buscador ni en la página web. Por el contrario, argumenta que provienen de la base de datos (BD), aportando evidencias y explicaciones técnicas. En uno de sus tuits el ciudadano expresa su preocupación por el uso de bases de datos corruptas en otros programas del gobierno: "lo que más preocupa es que estas bases de datos pueden estar también siendo usadas para dar más subsidios y ayudas estatales y revisando en diferentes lugares pude ver que hasta algunas de esas cédulas son de personas muertas, créditos en el icetex (sic) y hasta cotizan salud" (Díaz, 9 de abril 2020b).

Es justamente el imaginario alternativo sobre las bases de datos corruptas, manipuladas y de mala calidad uno de los más visibilizados cuando los ciudadanos explican sus experiencias con la página web de Ingreso 
Solidario y con otras páginas web de programas y entidades del gobierno, como la de la Registraduría, Sisben, Icetex y la Policía. Como consecuencia de la desactivación de la función de búsqueda en la página web de Ingreso Solidario, la auditoría colectiva se extiende a otras páginas del gobierno en donde es posible realizar consultas con números de identificación aleatorios y de personas muertas. Al ingresar números de cédula como 1111111111, $222222,44444,1212121212$ y 123456789 , por ejemplo, los ciudadanos encuentran en los resultados nombres de personas inexistentes como Cin Nus, Qwer qwer qwer, Millos David, GGG DERT y Nadie Nadie Nadie.

Las bases de datos del gobierno, de acuerdo con el imaginario que articulan los ciudadanos que participan en la auditoría colectiva, son descritas con adjetivos como podridas, sucias, desactualizadas y corruptas, y se las explica en función de su uso para robar, manipular y hacer trampa. Por ejemplo, un ciudadano dijo: "Y no se les cayó el sistema del \#IngresoSolidarioCorrupto, se les cayó la trampa y se develaron las podridas bases de datos con las que manipulan todo: auxilios, votaciones, ayudas" (Hernández, 10 de abril de 2020). Otro ciudadano explicó que, dada la baja calidad de la información de la base de datos, el sistema de información produce basura: “ $i C u a l$ (sic) error en un sencillo programa (no aplica software) de consulta de ayudas del gobierno? Si se consulta un archivo, o una base de datos trae lo que hay. Principio elemental de sistemas GIGO: Garbage in y garbage out. Eso tienen basura” (Rodríguez, 12 de abril de 2020).

Este imaginario alternativo y de resistencia refleja la profunda desconfianza de los ciudadanos en los sistemas de información del Estado y en la forma en que las tecnologías de clasificación y medición de la población son diseñadas y aplicadas por el gobierno colombiano. Las bases de datos del Estado, como archivos y registros de los ciudadanos, son imaginadas como tecnologías que se utilizan para diversos tipos de robo que van desde las elecciones hasta las ayudas a la población vulnerable. En este sentido, este imaginario sociotécnico apela también a la memoria colectiva sobre escándalos de fraudes electorales y desfalcos de la historia colombiana reciente. La creación y circulación del hashtag \#FraudeDeEstado durante la controversia sintetiza la fuerza de este imaginario, como podemos ob- 
servar en el siguiente tuit en \#FraudeDeEstado: "Lo que se demuestra con ingreso solidario, es una base de datos del estado que usan para desfalcos, para fraudes electorales, para robar y robar y robar. Es una mafia, una red de corrupción a gran escala que está en las instituciones, al más alto nivel” (Aldana, 9 de abril de 2020).

Ahora bien, como consecuencia de la intensa discusión en la esfera pública y de los procesos de resistencia algorítmica y contra la corrupción, el programa Ingreso Solidario tuvo que ser suspendido por el gobierno durante varios días. La aplicación de la segunda etapa fue retrasada, los giros a los beneficiarios se detuvieron y las páginas oficiales de varias entidades del Estado, incluyendo la de Ingreso Solidario, tuvieron que deshabilitar sus funciones de búsqueda. Además, el gobierno se vio obligado a verificar la base de datos del programa Ingreso Solidario y designó a la Registraduría Nacional para realizar este proceso.

Gracias a la oportunidad de exploración y experimentación con los sistemas de información del gobierno a través de buscadores en páginas web oficiales, las prácticas desarrolladas por ciudadanos durante la controversia de Ingreso Solidario desmontaron la opacidad de las tecnologías de clasificación y los programas antipobreza. Estas tecnologías son presentadas usualmente, en el imaginario sociotécnico dominante, como sistemas cerrados, con bases de datos y algoritmos que no pueden hacerse públicos por cuestiones de seguridad, control y privacidad. Las prácticas de búsqueda con números de identificación en la página web oficial de Ingreso Solidario abrieron, en cierta medida, una ventana en la caja negra de los sistemas de información del Estado, y permitieron a la ciudadanía experimentar su mal funcionamiento, encontrar evidencias de errores de inclusión y desarrollar una auditoría colectiva. De esta forma los ciudadanos del común lograron encontrarse con los sistemas de información, sus algoritmos y sus bases de datos, en situaciones concretas. Esa experiencia posibilitó la articulación y visibilización de imaginarios alternativos sobre el funcionamiento de esos sistemas y sus componentes. Como bien lo señala Bucher (2017) en su investigación sobre el imaginario algorítmico que elaboran los usuarios de Facebook, es gracias a este tipo de situaciones de encuentro con los 
sistemas de información que las personas toman conciencia de los algoritmos y les dan sentido de acuerdo con los sentimientos y afectos que genera la experiencia concreta.

En el caso de Ingreso Solidario, el encuentro de los ciudadanos con los sistemas de información se da en un contexto local de crisis, oposición al gobierno y protesta social. Ese contexto específico facilitó no solo la confluencia de la resistencia algorítmica y la resistencia a la corrupción a través de la auditoría colectiva a un programa social de administración de la pobreza, sino también la visibilización de imaginarios sociotécnicos alternativos sobre los sistemas de información del Estado y sus bases de datos.

\section{Conclusiones}

A partir del análisis de los casos que presenta este trabajo, surgen una serie de conclusiones asociadas, por un lado, con el desarrollo de problemáticas actuales en la actuación e interacción del Estado en los procesos de datificación de la pobreza y su relación con las formas corporativas transnacionales en busca de una supuesta eficacia y, por el otro, con la implicación histórica y gradual de estos mismos problemas en la base de las funciones que marcarán la institucionalidad de dichos Estados. En ese sentido, la teoría crítica latinoamericana viene arrojando, desde hace medio siglo, numerosas luces acerca de los caminos en que pueden derivar los diferentes procesos de registro y control de la pobreza, dependiendo de la construcción de las condiciones materiales y los imaginarios sociotécnicos asociados.

Tanto en el caso del programa Ingreso Solidario como en el del Sisben, la construcción de imaginarios sociotécnicos establecidos de un modo determinista en contextos del Norte Global, al ser aplicados, apropiados y trasladados a los contextos locales del Sur Global, tiene consecuencias inesperadas, con desviaciones y obstáculos que derivan de su falta de interés por la adaptación a las problemáticas locales, algo que ya advertían en la década de los setenta tanto los estudios sobre imperialismo cultural como aquellos centrados en la toma de decisiones en política tecnológica, en torno a los procesos de selección tecnológica y los acuerdos estructurales entre corporaciones transnacionales y Estado en América Latina para la administración pública de la información y la toma de decisiones. 
Las principales y más explícitas advertencias sintetizadas por las formas de teoría crítica latinoamericana se centraron en el cuestionamiento de los procesos de privatización de la administración de la información pública de los ciudadanos, así como en la importación -a través de la asimilación tecnológica- de esquemas, valores e imaginarios sociotécnicos ajenos a las necesidades y problemáticas de la realidad local. Ambos procesos derivarían, según dichas perspectivas, en la profundización de los esquemas de desigualdad, discriminación y corrupción que, supuestamente, pretendían solucionar. Ante la ausencia de propuestas políticas afines a sus planteamientos, unido a la profundización del modelo neoliberal de eficiencia e importación directa, los problemas presentados en el análisis de este trabajo dan la razón a la perspectiva crítica histórica y muestran, precisamente, las consecuencias directas de dicha profundización y la reducción de posibilidades de acción y resistencia.

No obstante, si bien existen modelos hegemónicos que han logrado establecerse como los imaginarios sociotécnicos dominantes, agregados en torno a la eficiencia y la neutralidad de la datificación, existen rupturas tácticas interesantes en forma de resistencias algorítmicas (herederas, a su vez, de procesos de resistencias culturales propios de la región) que transgreden dichas explicaciones institucionales y abren nuevamente los debates abandonados por las propuestas de la teoría crítica, construyendo nuevos imaginarios sociotécnicos alternativos que cuestionan y denuncian las bases corruptas, discriminatorias y desiguales de los Estados latinoamericanos. La utilización de formas propias de corrupción como modelos de acción táctica para el cumplimiento de sus necesidades derivará, a su vez, en el develamiento de los límites y las fallas de los modelos importados de datificación y reforzará la importancia de la localización de las soluciones, ampliando la capacidad de resistencia y sumando sectores poblacionales no necesariamente relacionados o afectados por dichas disfuncionalidades del sistema de administración.

En último lugar, el análisis llevado a cabo y la emergencia de un corpus de investigación definido acerca de los procesos de datificación en el Sur Global llevan a recomendar que se considere los imaginarios sociotéc- 
nicos locales, la corrupción de datos (Barreneche, 2019), la degradación de las bases de datos, vía manipulación maliciosa y desinformación, y el subsecuente mal funcionamiento de los sistemas algorítmicos asimilados como dimensiones centrales e indicadores relevantes a tener en cuenta en futuras investigaciones, pensadas desde esta perspectiva.

\section{Referencias}

Acevedo-Guerrero, T. (2019). Light is like water: flooding, blackouts, and the state in Barranquilla. Tapuya: Latin American Science, Technology and Society, 2(1), 478-494. DOI: https://doi.org/10.1080/ 25729861.2019.1678711

Aldana, O. [@oscaaldh] (9 de abril de 2020). \#FraudeDeEstado Lo que se demuestra con ingreso solidario, es una base de datos del estado que usan para desfalcos, para fraudes. Twitter. https://twitter. com/oscaaldh/status/1248283786711890000

Alexander, J. y Smith, J. (2010). Disinformation: A taxonomy. IEEE Security y Privacy, 9(1), 58-63. DOI: https://doi.org/10.1109/ MSP.2010.141

Arora, P. (2016). Bottom of the data pyramid: Big data and the global south. International Journal of Communication, 10, 19. Recuperado de: https://ijoc.org/index.php/ijoc/article/view/4297

Arteaga [@EpicuroDeSamos_] (10 de abril de 2020). A veces buscando plata se encuentra oro. Eso parece ser lo que pasó ayer con \#IngresoSolidario. Buscando saber. Twitter. https://twitter.com/EpicuroDeSamos_/status/1248293970087730000

Baczko, B. (1991). Los imaginarios sociales. Memorias y esperanzas colectivas. Buenos Aires: Nueva Visión.

Barreneche, C. (2019). Data corruption: The institutional cultures of data collection and the case of a crime-mapping system in Latin Ame- 
rica. Canadian Journal of Communication, 44(3). DOI: https://doi. org/10.22230/cjc.2019v44n3a3481

Beltrán, L. R. (1970). Apuntes para un diagnóstico de la incomunicación social en América Latina: la persuasión a favor del statu quo. Seminario Los Medios de Comunicación Colectiva y el Desarrollo Económico, Político y Social de América Latina, San José, Costa Rica, CEDAL.

Beltrán, L. R. (1978). Communication and cultural imperialism: USA-Latin American case. Media Asia, 24(1), 183-198.DOI: https://doi. org/10.1080/01296612.1978.11725945

Benjamin, R. (2020). Race after technology: Abolitionist tools for the new Jim Code [Book Review]. Social Forces, 98(4), 1-3. DOI: https:// doi.org/10.1093/sf/soz162

Borra, E. y Rieder, B. (2014). Programmed method: Developing a toolset for capturing and analyzing tweets. Aslib. Journal of Information Management, 66(3), 262-278. DOI: https://doi.org/10.1108/ AJIM-09-2013-0094

Bowker, G. C. y Star, S. L. (2000). Sorting things out: Classification and its consequences. Cambridge: MIT Press. DOI: https://doi.org/10.7551/ mitpress/6352.001.0001

Bucher, T. (2017). The algorithmic imaginary: Exploring the ordinary affects of Facebook algorithms, Information, Communication $y$ Society, 20(1), 30-44. DOI: https://doi.org/10.1080/136911 8X.2016.1154086

Cardoso, F. H. y Faletto, E. (1969). Dependencia y desarrollo en América Latina. México: Siglo XXI.

Castoriadis, C. (2013). La institución imaginaria de la sociedad. Barcelona: Tusquets. 
Chun, W. H. K. (2019). Queerying homophily. En Apprich, C., Chun, W. H. K., Cramer, F. y Steyerl, H. (eds.), Pattern discrimination. Minneapolis: University of Minnesota and Meson Press.

Colmenares, G. (1989). Relaciones e informes de los gobernantes de la Nueva Granada (vol. 1). Bogotá: Fondo de Promoción de la Cultura del Banco Popular.

Conpes (2016). Declaración de importancia estratégica del sistema de identificación de potenciales beneficiarios (Sisben IV). Documento No. 3877, Departamento Nacional de Planeación.

Dagnino, R., Thomas, H. y Davy, A. (1996). El pensamiento en ciencia, tecnología y sociedad en Latinoamérica: una interpretación política de su trayectoria. Redes, 7(3), 13-51.

Davis, A., Fenton, N., Freedman, D. y Khiabany, G. (2020). Media, democracy and social change. Re-imagining political communications. Londres: Sage.

DNP - Departamento Nacional de Planeación (2016a). Evaluación cualitativa a través de 24 grupos focales en seis regiones del país de la opinión de las personas incluidas en la base nacional, para complementar la evaluación del índice Sisben III. Recuperado de: https:// docplayer.es/5574528-Base-nacional-para-complementar-la-evaluacion-del-indice-sisben-iii.html

DNP - Departamento Nacional de Planeación (2016b). DNP alerta por nuevos 'colados' en el Sisben que ganan más de $\$ 3,8$ millones al mes. Recuperado de: https://www.dnp.gov.co/Paginas/DNPalerta-por-nuevos-\%E2\%80\%98colados\%E2\%80\%99-en-elSisb\%C3\%A9n.aspx

DNP - Departamento Nacional de Planeación (2020). Manual operativo del Programa Ingreso Solidario. 21 de marzo. Recuperado de: https:// 
ingresosolidario.dnp.gov.co/documentos/Manual_Operativo-Ingreso-Solidario.pdf

Díaz, Y. [@YilverDiaz_] (9 de abril de 2020a). Esta aplicación como la montaron lo único que hace es ir a usa $\mathrm{BD}$ a consultar si existe un usuario. Twitter. https://twitter.com/YilverDiaz_/status/1248266699008340000

Díaz, Y. [@YilverDiaz_] (9 de abril de 2020b). Lo que más preocupa es que estas bases de datos pueden estar también siendo usadas para dar más subsidios. Twitter. https://twitter.com/YilverDiaz_/status/ 1248266699008340000

Eubanks, V. (2018). Automating inequality: How high-tech tools profile, poli$c e$, and punish the poor. St. Martin's Press.

Fundación Karisma (2020). Experimentando con la pobreza: el Sisben y los proyectos de analítica de datos en Colombia. Recuperado de: https://web.karisma.org.co/wp-content/uploads/download-manager-files/Experimentando\%20con\%20la\%20pobreza.pdf

Gudynas, E. (2017). Extractivismos y corrupción en América del Sur. Estructuras, dinámicas y tendencias en una íntima relación, RevIISE, 10(10), 73-87. Recuperado de: https://dialnet.unirioja.es/ servlet/articulo?codigo $=6556726$

Gunder Frank, A. (1971). Lumpenburguesía: lumpendesarrollo. México: Era.

Hernández, H. G. [@jairohg] (10 de abril de 2020).Y no se les cayó el sistema del \#IngresoSolidarioCorrupto, se les cayó la trampa y se develaron las podridas bases. Twitter. https://twitter.com/jairohg/ status/1248639248560988162

Herrera, A. (1973). La creación de tecnología como expresión cultural. Nueva Sociedad, 8-9, 58-70. 
Jackson, S. J, Bailey, M. y Foucault Welles, B. (2020). \#нASHTAGACTIVISM: Networks of Race and Gender Justice. Cambridge: MIT Press. DOI: https://doi.org/10.7551/mitpress/10858.001.0001

Jasanoff, S. (2015a). Future imperfect: Science, technology and the imaginations of modernity. En Jasanoff, S. y Kim S-H. (eds.), Dreamscapes of modernity: Sociotechnical imaginaries and the fabrication of power (pp. 1-33). Chicago: University of Chicago Press. DOI: https:// doi.org/10.7208/chicago/9780226276663.001.0001

Jasanoff, S. (2015b). Imagined and invented worlds. En Jasanoff, S. y Kim S-H. (eds.), Dreamscapes of modernity: Sociotechnical imaginaries and the fabrication of power (pp. 321-342). Chicago: University of Chicago Press. DOI: https://doi.org/10.7208/chicago/9780226276663.001.0001

Kazansky, B. y Milan, S. (2021). Bodies not templates: Contesting dominant algorithmic imaginaries. New Media y Society, 23(2), 363-381. DOI: https://doi.org/10.1177/1461444820929316

Kim, S.-H. (2015). Social movements and contested sociotechnical imaginaries in South Korea. En Jasanoff, S. y Kim S-H. (eds.), Dreamscapes of modernity: Sociotechnical imaginaries and the fabrication of power (pp. 152-173). Chicago: University of Chicago Press.

Lander, E. (2004). Venezuela: la búsqueda de un proyecto contrahegemónico. En Ceceña, A. (comp.), Hegemonías y emancipaciones en el siglo XXI. Buenos Aires: Clacso.

Lehtiniemi, T. y Ruckenstein, M. (2019). The social imaginaries of data activism. Big Data y Society, 6, 1-12. DOI: https://doi. org/10.1177/2053951718821146

Lynch, N. (2020). Para una crítica de la democracia en América Latina. Buenos Aires: Clacso. DOI: https://doi.org/10.2307/j.ctv1gm0209 
Mansell, R. (2012). Imagining the Internet: Communication, innovation, and governance. Oxford: Oxford University Press.

Masiero, S. y Das, S. (2019). Datafying anti-poverty programmes: implications for data justice. Information, Communication \& Society, $22(7), 916-933$.

Marques de Melo, J. (1989). Comunicação na América Latina: desenvolvimento e crise. Sao Pablo: Papirus.

Mattelart, A. y Schmucler, H. (1983). América Latina en la encrucijada telemática. Barcelona: Paidós.

McFarlane, A. (1996). Political corruption and reform in Bourbon Spanish America. En Political corruption in Europe and Latin America (pp. 41-63). Londres: Palgrave Macmillan. DOI: https://doi. org/10.1007/978-1-349-24588-8_3

McPherson, T. (2018). Digital platforms and hate speech. Ponencia: Society for Cinema and Media Studies Conference Annual Conference, Toronto.

McQuillan, D. (2020). Deep bureaucracy and autonomist AI. En Hlavajova M. y Lütticken, S. (eds.), Deserting from the Culture Wars. Cambridge: MIT Press.

Medina, R. (20 de abril de 2020). Estadísticas de la situación digital de Colombia en el 2019 y 2020. Branch, Agencia de Marketing Digital Inbound. Recuperado de: https://branch.com.co/marketingdigital/estadisticas-de-la-situacion-digital-de-colombia-en-el2019-y-2020/

Mejías, U. y Couldry, N. (2019). Colonialismo de datos: repensando la relación de los datos masivos con el sujeto contemporáneo. Virtualis, 10(18), 78-97. Recuperado de: https://www.revistavirtualis. $\mathrm{mx} /$ index.php/virtualis/article/view/289 
Mignolo, W. D. (2003). Historias locales/diseños globales: colonialidad, conocimientos subalternos y pensamiento fronterizo. Madrid: Akal.

Milan, S. y Treré, E. (2019). Big data from the South (s): Beyond data universalism. Television y New Media, 20(4), 319-335. DOI: https:// doi.org/10.1177/1527476419837739

Morales, J. (1998). Mestizaje, malicia indígena y viveza en la construcción del carácter nacional. Revista de Estudios Sociales, 1, 39-43. DOI: https://doi.org/10.7440/res1.1998.05

Noble, S. U. (2018). Algorithms of oppression: How search engines reinforce racism. Nueva York: New York University Press. DOI: https://doi. org/10.2307/j.ctt1pwt9w5

Parente, E. (1979). O desafio da informação. Revista Nacional de Telecomunicações.

Pasquali, A. (1976). Comunicación y cultura de masas. Caracas: Monte Ávila.

Pérez Benavides, A. (2016). Estelas de trayectorias esparcidas: las tácticas indígenas en el contexto de las misiones. Colombia, 1880-1930. Memoria y Sociedad, 20(41), 43-53. DOI: https://doi.org/10.11144/ Javeriana.mys20-41.etet

Pérez, A. M. y Montoya Guzmán, J. D. (2010). La invención de la población: salud y riqueza en el Nuevo Reino de Granada, 1760-1810. Secuencia, 78, 15-42. Recuperado de: http://www.scielo.org.mx/ scielo.php?script=sci_arttextypid=S0186-03482010000300001

Pintos, J. (2015). Apreciaciones sobre el concepto de imaginarios sociales. Revista de Investigación Miradas, 13(1), 150-159. DOI: https://doi. org/10.22517/25393812.12281

Prieto, A. (1988). Crisis burguesa e imperialista en América Latina. La Habana: Editorial de Ciencias Sociales. 
O’Neil, C. (2016). Weapons of math destruction: how Big Data increases inequality and threatens democracy. Londres: Penguin.

Presidencia de la República (7 de abril de 2020). A partir de hoy, el Gobierno Nacional inicia la entrega del Ingreso Solidario a cerca de 3 millones de familias vulnerables, mayormente afectadas por la pandemia. Recuperado de: https://id.presidencia.gov.co/Paginas/ prensa/2020/Gobierno-Nacional-inicia-entrega-Ingreso-Solidario-cerca-de-3-millones-familias-vulnerables-afectadas-pandemia-200407.aspx

Rappaport, J. (2014). The disappearing mestizo: configuring difference in the colonial New Kingdom of Granada. Durham: Duke University Press.

Ricaurte, P. (2019). Data epistemologies, the coloniality of power, and resistance. Television y New Media, 20(4), 350-365. DOI: https:// doi.org/10.1177/1527476419831640

Rivera, D., Plata, J. y Rodríguez, J. (2018). Barómetro de las Américas Colombia 2018. Bogotá: Observatorio de la Democracia.

Rodríguez.R. [@gurozu] (12 de abril de 2020).¿Cual error en un sencillo programa (no aplica software) de consulta de ayudas del gobierno? Si se consulta un archivo. Twitter. https://twitter.com/guro$\mathrm{zu} /$ status/1249367140307730000

Solano, S. P. (2015). La construcción de los censos de población del Nuevo Reino de Granada a finales del siglo XVIII. El Taller de la Historia, 7(7), 41-99. DOI: https://doi.org/10.32997/2382-4794-vol.7num.7-2015-721

Steyerl, H. (2019). A sea of data: Pattern recognition and corporate animism. En Apprich, C., Chun, W. H. K., Cramer, F. y Steyerl, H. (eds.), Pattern discrimination. Minneapolis: University of Minnesota and Meson Press. 
Taylor, C. (2004). Modern social imaginaries. Durham: Duke University Press. DOI: https://doi.org/10.1215/9780822385806

Treré, E. (2018). From digital activism to algorithmic resistance. En Meikle, G. (ed.), The Routledge Companion to Media and Activism (pp. 367-375). Londres, Nueva York: Routledge. DOI: https://doi. org/10.4324/9781315475059-39

Vaitsos, C. (1971). Opciones estratégicas en la comercialización de tecnología: el punto de vista de los países en desarrollo. Comercio Exterior, XXI(9), 803-815. Recuperado de: http://revistas.bancomext. gob.mx/rce/magazines/619/8/CE_SEPTIEMBRE_1971_.pdf

William Cardona, Canal Oficial (s.f.). El Sisben y la trampa. Canal YouTube: https://www.youtube.com/watch?v=e-zobqX3Lh4ylc=Ugh LbuAGzCX4EngCoAEC 